\title{
MUTATIONS ALTERING SYNAPTIC CONNECTIVITY BETWEEN IDENTIFIED NEURONS IN DROSOPHILA ${ }^{1}$
}

\author{
JOHN B. THOMAS ${ }^{2}$ AND ROBERT J. WYMAN \\ Department of Biology, Yale University, New Haven, Connecticut 06511 \\ Received June 27, 1983; Accepted August 23, 1983
}

\begin{abstract}
By studying the effects of mutations on a simple circuit of identified neurons in Drosophila, we have found genes whose proper functioning is necessary to produce normal synaptic connections between the neurons. These neurons comprise the giant fiber (GF) system; the GFs are command neurons activated by a light-off stimulus and evoke a stereotyped pattern of activity in the thoracic muscles producing an escape jump. Each GF monosynaptically drives a motor neuron innervating the tergotrochanteral muscle (jump muscle, TTM). Each GF also disynaptically drives the motor neurons innervating the dorsal longitudinal flight muscle (DLM) via the peripherally synapsing interneuron (PSI) (King, D. G., and R. J. Wyman (1980) J. Neurocytol. 9: 753-770; M. A. Tanouye and R. J. Wyman (1980) J. Neurophysiol. 44: 405-421). A search was made for mutations affecting these identified synapses. Fifty thousand mutagenized flies were screened for nonjumping behavior to the light-off stimulus. Fifty-seven nonjumping mutant lines were established from individuals selected in the screen. Members of the lines were then tested for abnormal GF motor output to the TTM and DLM. From these lines, four X-linked mutations (representing three complementation groups) were isolated which affect the circuit. The mutations differentially disrupt specific synapses within the GF system. One mutation, bendless, disrupts synaptic transmission between the GF and the TTM motor neuron. Another, gfA, disrupts the synaptic connections of the PSI, and a third mutation, passover, disrupts transmission in both pathways.
\end{abstract}

A central concern of developmental neurobiology is to understand the mechanisms by which nerve cells form specific connections. Most efforts at solving this problem involve describing the sequence of events during normal development or after surgical intervention. An alternative approach, which we use, is to search for genes whose normal functioning is necessary for the establishment of proper neuronal connections. If such genes were identified, then it should be possible, using recombinant DNA technology, to identify the gene products. Thus, for instance, if surface-bound molecules function as guidance cues during axon growth and choice of synaptic targets, then the genes and gene products of either the molecules themselves (if proteins) or the enzymes involved in their synthesis (if not proteins) might be identified.

Mutations which alter genes involved in specifying neural connections would be expected to alter the normal

\footnotetext{
${ }^{1}$ This work was supported by United States Public Health Service Grants NS-07314 and NS-14887. We wish to thank Ms. Catherine Blackmon for her unfailing patience with the manuscript.

${ }^{2}$ To whom all correspondence should be sent, at his present address: Department of Biological Sciences, Stanford University, Stanford, CA 94305.
}

pattern of connectivity and to disrupt the behavior generated by those connections. Many mutations in Drosophila exist which affect various components of the fly's behavioral repertoire. Mutations have been isolated which affect phototaxis, flying, optomotor behavior, chemosensory behavior, circadian rhythms, learning, and memory (for review, see Hall, 1982). However, due to the complexity of the neuronal circuits producing these behaviors, it has not been possible to determine if these mutations cause changes in the connectivity. To overcome this difficulty we have chosen a simple network of neurons in Drosophila, the giant fiber (GF) system, in which we can test for the functioning of identified synaptic connections and in which we can select for mutations which disrupt these connections.

The giant fibers are a bilateral pair of large $(5 \mu \mathrm{m})$ interneurons descending from the brain to the thoracic ganglion. Their wild-type morphology has been described by Koto et al. (1981) using intracellular injection of Lucifer Yellow. The GF cell bodies are located in the brain and send processes into visual and antennal centers. The axon of each GF descends along the dorsal midline of the cervical connective to the thoracic ganglion. Within the mesothoracic neuromere the GF makes 
synaptic contact with two identified neurons (Fig. 1a) (King and Wyman, 1980). Near the middle of the neuromere, the GF synapses with the peripherally synapsing interneuron (PSI) which crosses the ganglion and chemically synapses with the five motor neurons innervating the contralateral dorsal longitudinal flight muscle (DLM). The GF then bends laterally and at its terminus contacts the central process of a motor neuron innervating the ipsilateral tergotrochanteral muscle (TTM, or jump muscle). In EM sections of these regions King and Wyman (1980) found evidence of gap junctions between the GF and the PSI and between the GF and the TTM motor neuron.

Tanouye and Wyman (1980) have shown that extracellular stimulation of the GFs in the brain evokes a characteristic pattern of activity in the TTM and DLM. Both muscles are driven with short and constant latencies, and the pathway to each muscle is extremely stable. Based on latency analysis of these pathways, they concluded that transmission between the GF and TTM motor neuron and also between the GF and PSI was electrical, thus complementing the anatomical findings of King and Wyman (1980).

In this paper we show that the giant fibers mediate an escape jump response to a visual stimulus. By screening for nonjumping behavior we have isolated mutations induced on the $\mathrm{X}$ chromosome which disrupt proper functional connectivity between the identified neurons driving this response.

\section{Materials and Methods}

Stocks. Except where noted, descriptions of mutations used in this study can be found in Lindsley and Grell (1968). Wild-type strains used were Canton-S (C-S) and Hochi. Four white-eyed strains were used: white $(w)$, cinnabar brown (cn bw), vermilion; brown $(v ; b w)$, and brown;scarlet $(b w ; s t)$. Nonjumping mutations were mapped by meiotic recombination with the markers yellow, crossveinless, vermilion, forked, and carnation. A series of X chromosome deficiencies and translocations was used for cytological mapping of the mutations: $D f(1) J A 27$ (Campos-Ortega and Jimez, 1980), $D f(1) 16$ 3-22 (Schalet and Lefevre, 1976), Df(1)HA92 (CamposOrtega and Jimez, 1980), and $T(1 ; 4) B^{\mathrm{s}}$. The deficiencies were isolated by Dr. G. Lefevre. All crosses were reared at $25^{\circ} \mathrm{C}$.

Mutagenesis. One- and 2-day-old bw;st males were fed the mutagen ethyl methane sulfonate (EMS) according to the method of Lewis and Bacher (1968) and mated with $C(1) M 3 / Y$ females also carrying bw;st. $\mathbf{F}_{1}$ male progeny from this cross inherit the mutagenized paternal $\mathrm{X}$ chromosome and express mutations induced on it. These $F_{1}$ males were screened for nonjumping behavior; individual C(1)M3 lines were established from the nonjumpers selected in the behavioral screen.

Behavioral screen. The screening device was placed in a box lined with white paper. Illumination of $700 \mathrm{Lux}$ was provided by a fluorescent lamp positioned above the box; a translucent cover was placed on top of the drum to diffuse the light. A brief flicker of the lamp served as the light-off stimulus and was produced by driving a solenoid-activated relay in series with the $117-\mathrm{V}$ line.
Stimuli were delivered at 15 -sec intervals unless otherwise noted.

Muscle recordings. Flies were tethered to an insect pin using Eastman 910 contact cement (Kodak Chemicals). A pair of insulated tungsten electrodes was inserted into the brain for extracellular stimulation of the GFs or into the thoracic ganglion for extracellular stimulation of the motor neurons. Short stimulus durations $(0.05 \mathrm{msec})$ were used to facilitate latency measurements. Recording electrodes (insulated tungsten) were placed intracellularly in a fiber of the DLM and in the TTM. An uninsulated tungsten electrode inserted in the abdomen served as a ground. All experiments were carried out at $23^{\circ} \mathrm{C}$.

GF recordings. Techniques of GF recordings and dye injection were modified from Tanouye and Wyman (1980) and Koto et al. (1981). Flies were mounted ventral side up within a small hole at the bottom of a $2 \times 2 \times$ $0.2 \mathrm{~cm}$ plastic dish. They were sealed in wax such that the abdominal and thoracic spiracles were exposed to an air flow while the ventral nerve cord could be bathed in saline. Removal of the prothoracic pre-episternum provided access to the cervical connective where the giant fibers were intracellularly penetrated. Insulated tungsten stimulating and recording electrodes were inserted in the brain and TTM and DLM muscles. Microelectrodes were filled with either $3 \mathrm{M} \mathrm{KCl}$ or with a $5 \%$ solution of Lucifer Yellow (backfilled with $0.5 \% \mathrm{LiCl}$ ). Dye was injected for 2 to $10 \mathrm{~min}$ using $10 \mathrm{nA}$ hyperpolarizing pulses. Intracellular penetration of the GF could be confirmed by virtue of its having the same spiking threshold as the DLM to stimulation in the brain (Tanouye and Wyman, 1980). In dye injection experiments the GF could be identified on the basis of its characteristic morphology and position within the cervical connective.

After dye injection animals were placed in 5\% formaldehyde (phosphate buffered to $\mathrm{pH} 7$ ) where they were left for 1 to $12 \mathrm{hr}$. In the fixative the brain and thoracic ganglion were dissected out, dehydrated in an alcohol series, and cleared for whole mount observation in methyl benzoate. For sectioning, the tissue was embedded in Spurr's medium. Whole mount and sectioned materials were viewed with fluorescence optics as described by Stewart (1978).

\section{Results}

The giant fibers drive a stereotyped escape response. Flies will jump to a variety of stimuli. When tactile and visual stimuli were delivered to a tethered fly while recording activity from the TTM and the DLM, it was found that different stimuli elicited different patterns of activation of the thoracic muscles. In general, repetition of the same stimulus evoked variable responses. To determine which, if any, of these responses were driven by the giant fiber, the pattern of muscle response to giant fiber stimulation was studied. The giant fiber was intracellularly stimulated in the cervical connective. It was found that the GF drove the TTM and DLM with very constant latencies of $0.9 \mathrm{msec}$ for the TTM and $1.3 \mathrm{msec}$ for the DLM (Fig. 1b).

Tanouye and Wyman (1980) found the same pattern of response when the GFs were extracellularly stimulated 

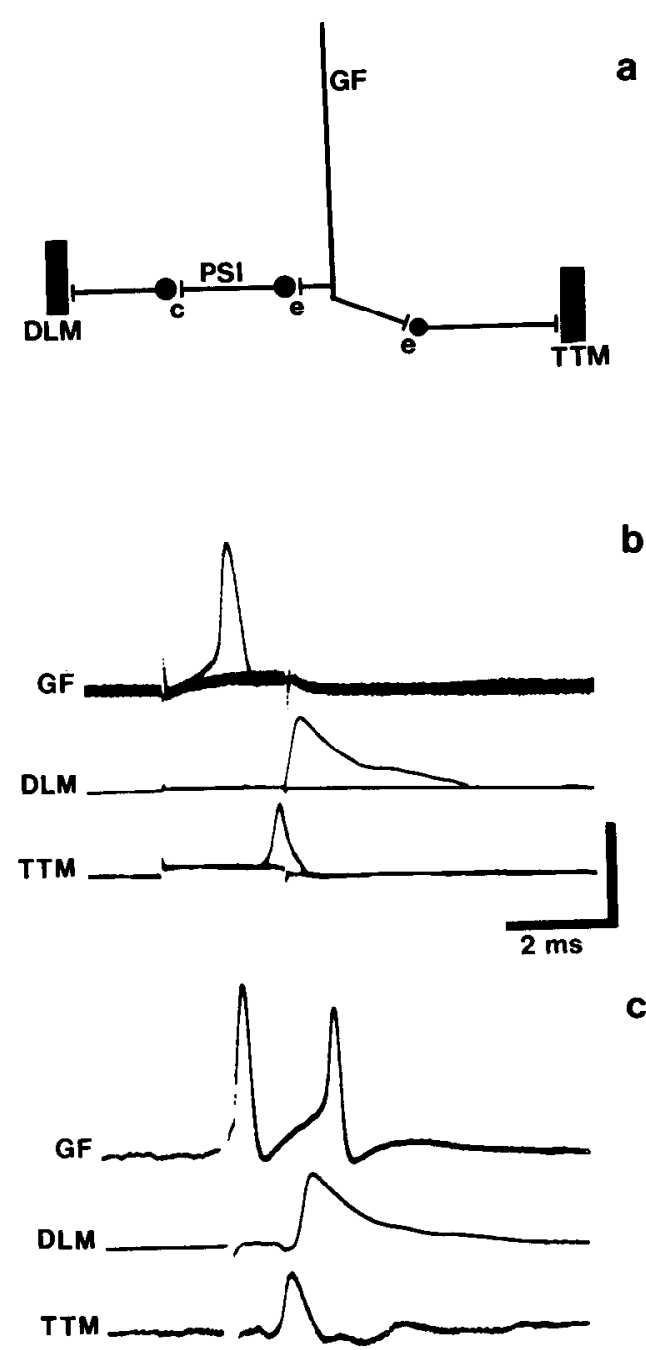

Figure 1. Giant fiber motor output in wild-type. $a$, Schematic diagram showing the synaptic relationship among identified neurons of the GF system. $e$, electrical synapse; $c$, chemical synapse. Modified from King and Wyman (1980) and Tanouye and Wyman (1980). $b$, Intracellular stimulation of the GF while recording muscle responses in the TTM and DLM. Two current pulses at threshold for the GF spike were delivered: one pulse was ineffective, the other elicited spikes in the GF, the DLM, and the TTM. Injected current was $3 \mathrm{nA}$; the bridge was intentionally overbalanced for display purposes. Vertical calibration is $40 \mathrm{mV}$ for GF and DLM, $10 \mathrm{mV}$ for the TTM. $c$, Extracellular stimulation of the GF in the brain with concomitant recording of the GF, TTM, and DLM. Spikes in all three elements are elicited at the same threshold, and the muscle response latencies (measured from the first GF spike) are identical to those seen in $b$. Vertical calibration is the same as in $b$.

in the brain. In the present study, for comparison with the mutants, the GF's were stimulated extracellularly in 20 wild-type Canton-S (C-S) flies and the TTM and DLM response latencies recorded. The result from one individual is shown in Figure 1c. The spikes in the GF, TTM motor neuron, and DLM all had the same threshold, and the latency intervals between the three were indistinguishable from those seen in the intracellular stimulation experiment. The pooled data from the 20 individuals are shown in Table I. The mean TTM latency was $0.88 \pm 0.09 \mathrm{msec}$, and the mean DLM latency was $1.30 \pm 0.09 \mathrm{msec}$. The mean latency varied somewhat with temperature, but at any given temperature the response was always stereotyped with standard deviations of less than $0.1 \mathrm{msec}$.

Of all the stimuli used to elicit jump responses, only a rapid light-off stimulus elicited this stereotyped pattern of activation of the GF, TTM, and DI.M. Figure $2 a$ shows an intracellular recording of a GF in an individual presented with the light-off stimulus. The GF spike occurred $20 \mathrm{msec}$ after the stimulus and elicited the characteristic pattern of activity in the TTM and DLM (Fig. 2b). Latencies from the GF spike to the TTM and DLM responses were indistinguishable from those

TABLE I

Muscle response latencies of the mutants

\begin{tabular}{lccccc}
\hline \multirow{2}{*}{ Genotype } & \multicolumn{2}{c}{$\begin{array}{c}\text { Latency to GF Stimulation } \\
(\mathrm{msec})^{a}\end{array}$} & & \multicolumn{2}{c}{$\begin{array}{c}\text { Latency to Motor Neuron } \\
\text { Stimulation (msec) }\end{array}$} \\
\cline { 2 - 3 } \cline { 5 - 6 } & TTM & DLM & & TTM & DLM \\
\hline C-S $^{c}$ & $0.88 \pm 0.09$ & $1.30 \pm 0.09$ & & $0.63 \pm 0.04$ & $0.65 \pm 0.05$ \\
bendless & $2.31 \pm 0.44$ & $1.29 \pm 0.10$ & & $0.63 \pm 0.05$ & $0.64 \pm 0.06$ \\
passover & $1.50 \pm 0.18$ & Not driven & & $0.62 \pm 0.06$ & $0.63 \pm 0.04$ \\
gfA & $0.87 \pm 0.09$ & $3.21 \pm 0.94$ & & $0.62 \pm 0.04$ & $0.66 \pm 0.06$ \\
\hline
\end{tabular}

${ }^{a} N=20$ for each genotype.

${ }^{b} N=10$ for each genotype.

${ }^{c}$ cf. Tanouye and Wyman (1980) for wild-type values.

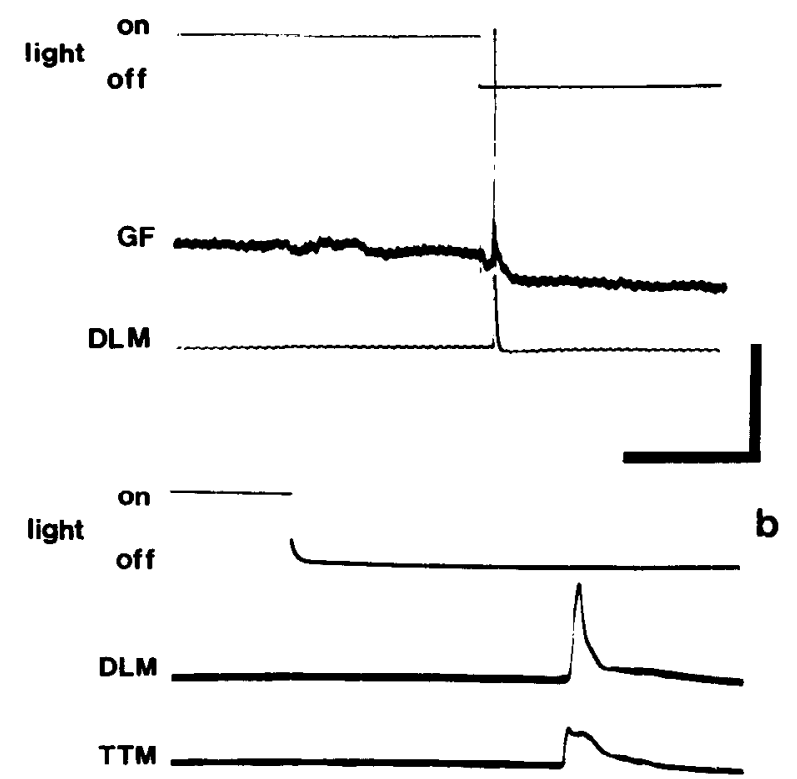

Figure 2. GF activation by a light-off stimulus. $a$, Intracellular spike in the GF evoked by the light-off stimulus in a $b w$;st individual. The DLM spike had the same threshold as the GF spike. Latency from the light-off stimulus to the GF spike is $20 \mathrm{msec}$ with a further $1.3 \mathrm{msec}$ to the DLM spike. Time calibration is $200 \mathrm{msec}$; vertical calibration is $20 \mathrm{mV}$ for GF, $40 \mathrm{mV}$ for DLM. $b$, Expanded time base record of the response in the TTM and DLM to the light-off stimulus in bw;st. The pattern of muscle responses is identical to that seen in the GF stimulation experiments shown in Figure 1. Time calibration is 10 msec; vertical calibration is $100 \mathrm{mV}$ for the DLM and 40 $\mathrm{mV}$ for the TTM. The top trace in $b$ and $c$ is the voltage record of the stimulator output driving the electronic shutter. 
evoked by intracellular stimulation of the GF. In tethered flies, other stimuli showed different patterns of activation of the TTM and DLM and thus likely activate the muscles by pathways that do not involve the giant fiber.

Since the TTM is the main extensor of the mesothoracic leg which provides the majority of thrust during a jump (Mulloney, 1969), activation of the GFs should result in a jump. When presented with the light-off stimulus, an unrestrained fly was found to exhibit an abrupt jump response. The probability of eliciting this jump response in wild-type flies was rather low: Hochi and C-S strains responded to only $34 \%$ and $37 \%$ of the stimuli, respectively. White-eyed flies jumped with greater reliability. Four genotypes conferring white eyes were tested: $w$ (responding to $58 \%$ of stimuli), $v ; b w(60 \%)$, cn bw (72\%), and bw;st (97\%). Since all four of the whiteeyed strains (each carrying a different combination of mutations) showed an enhancement of the response, it is probable that the reduction in screening pigments in these mutants per se, rather than pleiotropic effects of the particular mutations, is responsible for the increased responsivity. In addition, GF motor output in each of the white-eyed strains was indistinguishable from wild-type, and the GF morphology in three of the strains examined has been shown to be completely normal (Koto et al., 1981; Thomas and Wyman, 1982).

In summary, the giant fiber drives a very rapid and stereotyped response. Activation of the giant fiber and the ensuing stereotyped response are elicited by visual stimuli, resulting in a jump. The jumping reliability in the $b w$;st strain is great enough to allow the efficient screening of large numbers of flies for nonjumping mutants.

Giant fiber output in existing mutations. Before searching for induced mutations affecting the giant fiber system, the physiological output of the giant fiber was tested in a variety of existing mutations known or suspected to alter either the morphology or physiology of the nervous system. Five individuals for each genotype were tethered and the GF stimulated extracellularly in the brain while recording from the TTM and DLM. Table II lists the mutations tested along with their map position and phenotypes. In none of these mutations was the pattern of evoked responses in the TTM and DLM different from wild-type.

Behavioral screen. The screening device for selecting nonjumping flies consisted of a $250-\mathrm{ml}$ boiling flask inverted in a 250-ml beaker. Flies were placed inside the flask where they were free to walk along its inner surface. A series of light-off stimuli were delivered, causing them to jump and fall a small distance. After several stimuli, jumpers fell into the beaker from which they were unable to reenter the testing flask. The three or four remaining flies could then be tested individually and those capable of jumping eliminated.

The efficiency of the screening procedure was tested by placing sets of $100 \mathrm{bw}$;st males plus a single fly carrying the $B a r$ mutation into the testing flask. Bar flies have severely reduced numbers of ommatidia and do not jump to the light-off stimulus. Light-off stimuli were delivered every $15 \mathrm{sec}$. At the end of $3 \mathrm{~min}$ it was noted whether the Bar fly had remained in the testing area. Ten trials were run using separate sets of flies for each trial. In 9 of the 10 trials the Bar fly remained. The screening protocol was both selective (flies capable of jumping were always eliminated) and efficient (90\% of true nonjumpers are recovered from the screen). The rate of screening was quite rapid, allowing the handling of large numbers of mutagenized flies.

Fifty thousand $F_{1}$ males carrying mutagenized $X$ chromosomes (see "Materials and Methods") were screened for nonjumping behavior to the light-off stimulus. Of these, 332 failed to jump. These males were mated individually to $C(1) M 3 / Y$ females to establish lines. In these

TABLE II

Existing mutations tested for abnormal giant fiber motor output

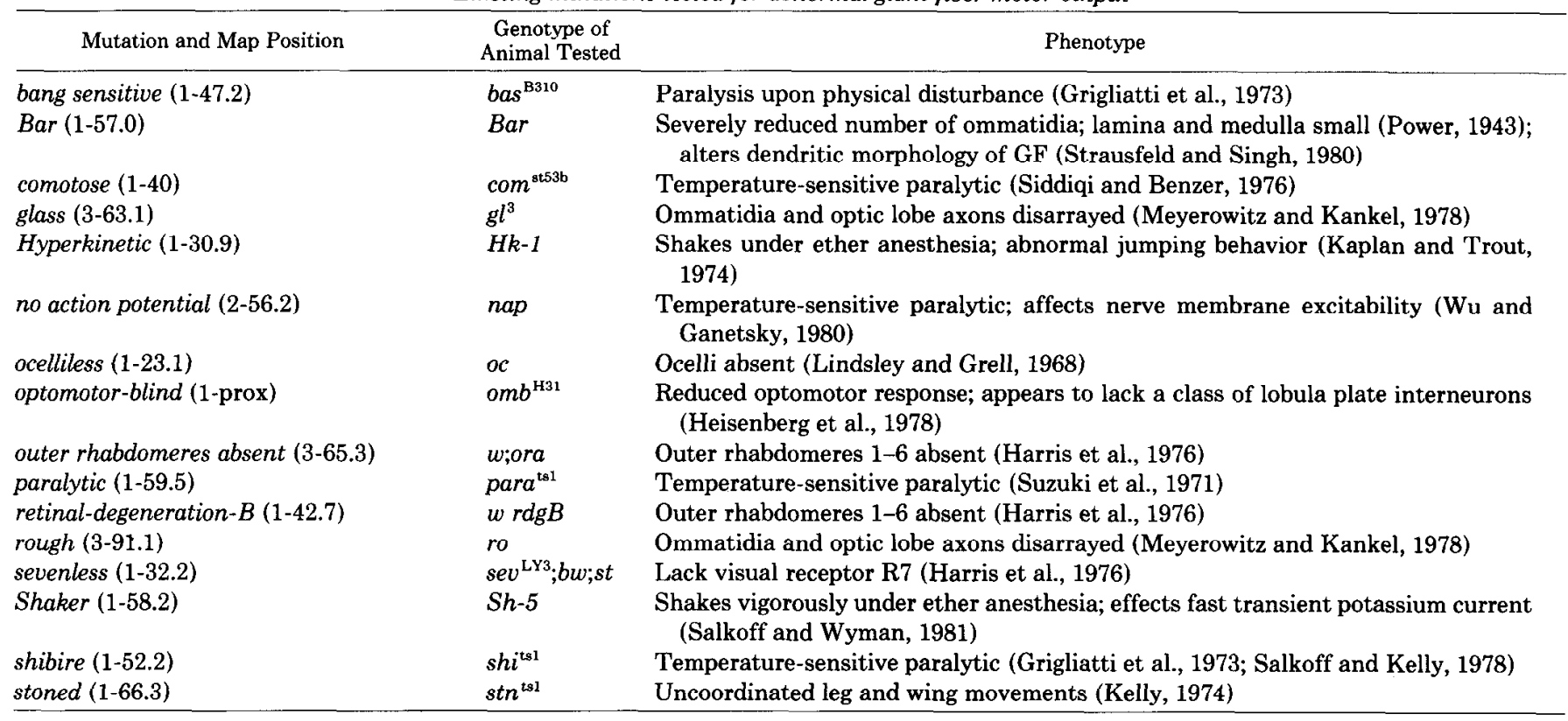


lines the mutagenized X chromosome is inherited exclusively from father to son. The male progeny from each of these lines were retested. Of the original 332 lines, 198 were capable of jumping and were discarded, 77 produced either no males or no progeny at all, and the remaining 57 lines were nonjumping. These nonjumping lines consisted of two classes: 41 totally nonjumping lines in which every male failed to jump to the light-off stimulus, and 16 partially nonjumping lines in which $20 \%$ to $90 \%$ of the males failed to jump.

Electrophysiological screen. The inability to jump in these 57 mutant lines could have a variety of causes including defects presynaptic to the giant fibers (e.g., in the visual system), in the circuit of identified cells itself or in the musculoskeletal output elements. Since we were primarily interested in mutations that affeet the giant fibers and associated identified neurons, we tested mutant males from each line for abnormal TTM and DLM response to extracellular stimulation of the GFs. Nonjumping mutants that exhibited normal GF output were presumed to have visual system or other defects presynaptic to the GF. We could detect mutants with defective muscular or skeletal elements by extracellularly stimulating the motor neurons in the ganglion with concomitant monitoring of muscle and leg responses.

The nonjumping males from the 16 partially nonjumping lines all showed normal giant fiber output. Among the 41 totally nonjumping mutant lines, 36 showed normal output and one (nj42, not discussed further here) proved to be missing the TTM muscle (Thomas, 1980; Costello and Thomas, 1981). The four remaining lines showed abnormal GF output. These four mutations comprise three X-linked complementation groups: bendless, passover, and $g f A$ (two alleles, $g f A^{1}$ and $g f A^{2}$ ). The physiological phenotypes of the two $g f A$ alleles are indistinguishable from each other. Each of the four mutations is recessive and shows complete penetrance of both the nonjumping behavior and the physiological phenotype.

bendless (ben). Figure $3 a$ shows the response in the GF, TTM, and DLM of a bendless individual to extracellular stimulation of the GFs in the brain. In these experiments, Lucifer Yellow fills were used to confirm that the intracellular electrode was in the giant fiber. It can be seen that the DLM latency is normal, but the TTM latency is $2.5 \mathrm{msec}$, or more than twice the normal latency. Intracellular stimulation of the GF evoked the same abnormal response. The pooled results for 20 ben flies are shown in Table I. In all flies examined, the DLM response latency and following frequency characteristics were indistinguishable from wild-type. However, the TTM response latency was longer and more variable with a mean latency of $2.31 \pm 0.44 \mathrm{msec}$ in contrast to wild-type where the mean latency was $0.88 \pm 0.09 \mathrm{msec}$.

In wild-type the TTM can be driven by the GF at frequencies exceeding $100 \mathrm{~Hz}$ (Tanouye and Wyman, 1980). However, in ben flies, attempts to drive the TTM at frequencies of $1 \mathrm{~Hz}$ or higher resulted in failure of response to many stimuli. Figure $3 b$ shows these interspersed failures to a stimulus train at $2 \mathrm{~Hz}$. To eliminate the possibility that the low following frequency and abnormally long latency in the mutant were due to defects in TTM motor neuron conduction or neuromus-
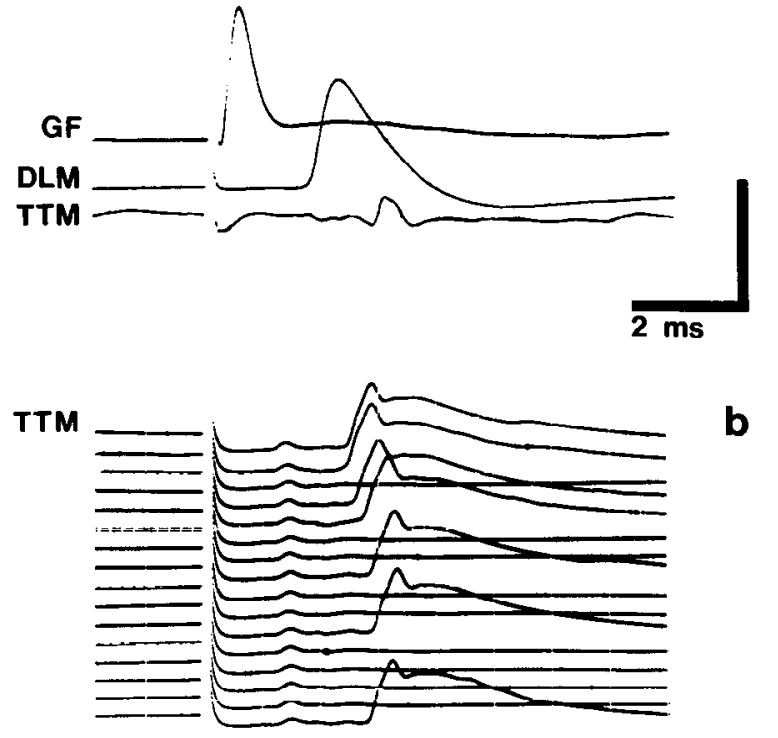

b

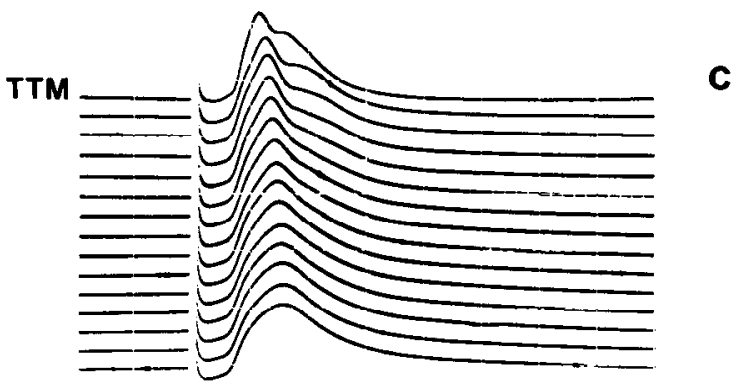

Figure 3. GF motor output in bendless. a, Extracellular stimulation of the GF, recording from the GF, TTM, and DLM. DLM response latency is normal $(1.4 \mathrm{msec})$, but the TTM latency is abnormally long ( $2.5 \mathrm{msec})$. GF penetration was confirmed by intracellular injection of Lucifer Yellow. Vertical calibration is $40 \mathrm{mV}$ for GF and DLM, $20 \mathrm{mV}$ for TTM. $b$, Response in the TTM of ben to stimulation of the GF at $2 \mathrm{~Hz}$. The first in a train of 16 stimuli is at the top, the last is at the bottom. The TTM failed to 9 of the 16 stimuli. Vertical calibration is $20 \mathrm{mV}$. $c$, Response in the TTM of ben to extracellular stimulation of its motor neuron at $50 \mathrm{~Hz}$. Muscle responses to the train are displayed as in $b$. The TTM responded without a failure. Vertical calibration is $20 \mathrm{mV}$.

cular transmission, the TTM motor neuron was stimulated extracellularly in the ganglion. The results are shown in Figure $3 c$ and Table I. Both the following frequency and synaptic delay of the TTM neuromuscular junction in all ben flies examined were normal. The mean latency from stimulus to TTM response was $0.63 \pm 0.05$ msec, and the junction followed stimulation frequencies in excess of $100 \mathrm{~Hz}$. This shows that the defect in the pathway responsible for the abnormal TTM response lies not in TTM motor neuron conduction nor at the neuromuscular junction but at the GF-TTM motor neuron junction.

Genetics of bendless. bendless maps between vermilion and forked on the $\mathrm{X}$ chromosome meiotic map. Hetero- 
zygous ben/+ females show normal phenotype. However, the mutant phenotype is expressed in females heterozygous for ben and the $\mathrm{X}$ chromosomal deletion Df(1)HA92. This deletion has been shown cytologically to have breakpoints in bands 12A6-7 and in 12D3 (and to be missing the bands in between) on the salivary gland chromosome map. Thus, the ben mutation is located between these breakpoints.

passover (pas). Figure $4 a$ shows the response evoked in the TTM and DLM of a passover individual to extracellular stimulation of the GF in the brain. Neither muscle response is normal. In all flies examined the TTM responses had abnormally long latencies (mean latency $=1.50 \pm 0.18 \mathrm{msec}$, Table I), and no response could be evoked in the DLMs. The same response was evoked by intracellular stimulation of the GF. The GF-TTM pathway in pas is also more labile than normal; the TTM can be driven $1: 1$ by the giant fiber only at frequencies less than $1 \mathrm{~Hz}$. Figure $4 b$ shows the response in a TTM of a pas individual to extracellular stimulation of the GF at $2 \mathrm{~Hz}$. At this frequency the TTM failed to many stimuli.

To eliminate the possibility that the lack of response in the DLM and the abnormal response in the TTM were due to defects in the motor neurons or neuromus-

a

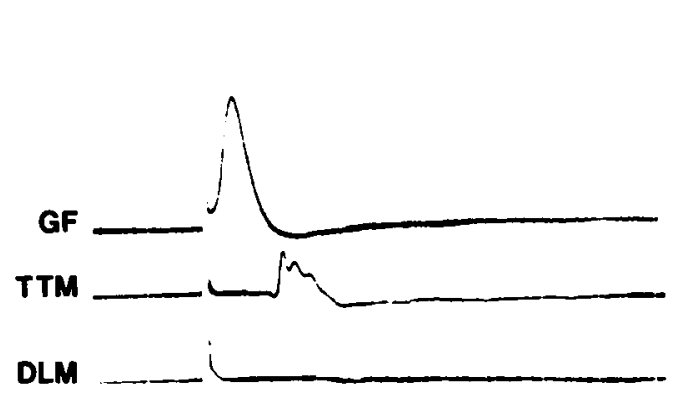

cular junctions, the motor neurons were stimulated extracellularly in the ganglion while recording from the muscles. Figure 4, $c$ and $d$ and Table I show the results of these experiments. Both the TTM and DLM showed normal latencies and following frequencies to ganglion stimulation, indicating that the defects lie centrally, between the GF and the TTM motor neuron (for the GFTTM pathway) and between the GF and the DIM motor neurons (for the GF-DLM pathway).

Genetics of passover. passover maps proximally to forked on the X chromosome and was originally called nj156 (Thomas, 1980). Females heterozygous for pas and $D f(1) 16-3-22$ express the pas physiological phenotype. On this evidence, pas is located cytologically between bands 19D1 and 20A2 on the polytene chromosome map. Hyperploid males homozygous for pas, but carrying, in addition, an extra proximal segment of the $\mathrm{X}$ chromosome from $T(1 ; 4) B^{\mathrm{s}}$ (which includes these bands), do not express the pas phenotype.

gfA. GF output in $g f A^{1}$ is shown in Figure $5 a$. Both the TTM and the DLM response thresholds are the same as the giant fiber spiking threshold to brain stimulation, and the muscles could be driven by intracellular stimulation of the GF. In this mutant the TTM is driven
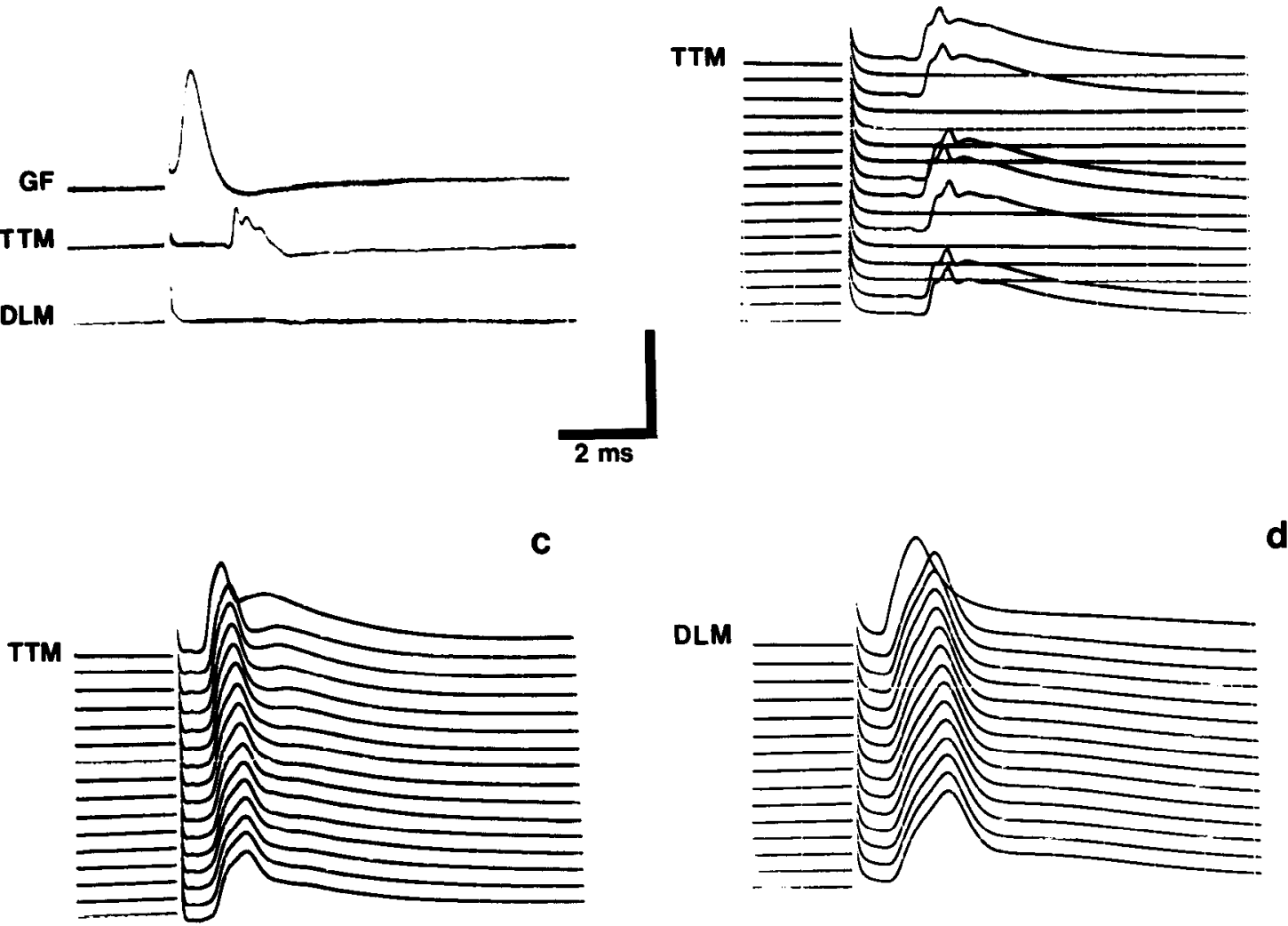

Figure 4. GF motor output in passover, a, Extracellular stimulation of the GF in pas with concomitant recording of GF, TTM, and DLM. Both muscle responses are abnormal: the DLM is not driven at all; the TTM latency is abnormally long at 1.2 msec. $\mathrm{GF}$ penetration was confirmed by Lucifer Yellow injection. Vertical calibration is $40 \mathrm{mV}$ for GF and DLM, $20 \mathrm{mV}$ for TTM. $b$, Response in the TTM of a pas individual to stimulation of the GF at $2 \mathrm{~Hz}$. The top trace is the first of a train of 16 stimuli, the bottom trace is the last. The 'I"I'M muscle failed to nine of the stimuli. Vertical calibration is $20 \mathrm{mV}$. $c$, Response of the TTM of pas to motor neuron stimulation at $50 \mathrm{~Hz}$. The muscle responded to all stimuli without a failure. Vertical calibration is $20 \mathrm{mV}$. $d$, Response in a DLM fiber to stimulation of its motor neuron at $50 \mathrm{~Hz}$. The muscle responded to all stimuli without a failure. Calibration is $40 \mathrm{mV}$. The trace displays in $c$ and $d$ are the same as in $b$. 
a
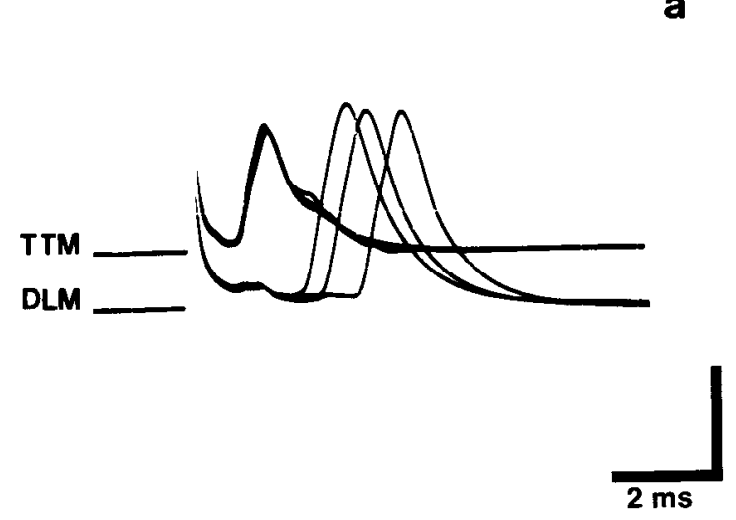

C

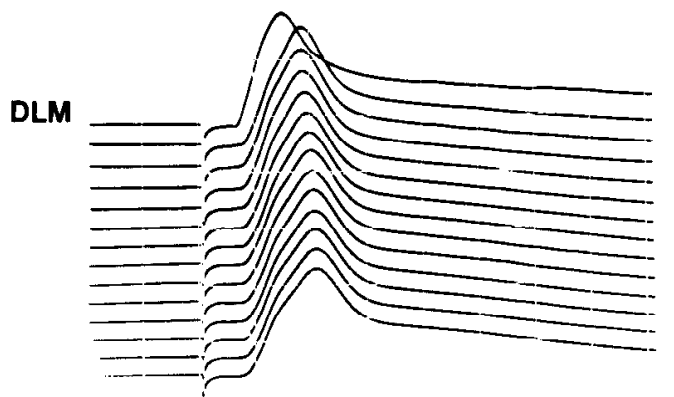

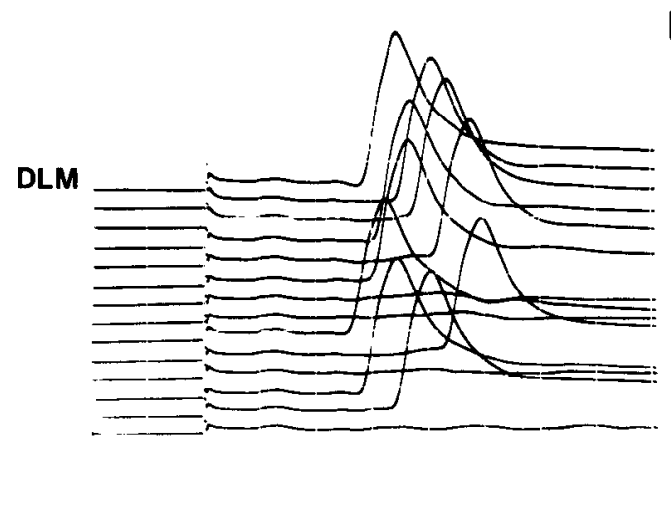

b

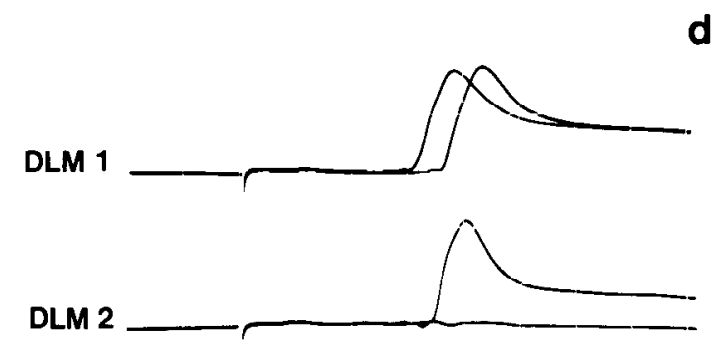

Figure 5. GF motor output in $g f A^{1}$. a, The response in the TTM and DLM to extracellular stimulation of the GF. Three stimuli were delivered. The TTM responded with normal latency $(0.9 \mathrm{msec})$, but the DLM response latency is long and variable. Vertical calibration is $20 \mathrm{mV}$. $b$, Response in a DLM fiber to GF stimulation at $2 \mathrm{~Hz}$. The first stimulus of the train is at the top. The DLM response latency is long and variable and failed to 4 of the 14 stimuli. $c$, Response in a DLM fiber to stimulation of its motor neuron at $50 \mathrm{~Hz}$. It followed without a failure. The trace display is the same as in $b$. $d$, Response in two ipsilateral DLM fibers to GF stimulation. Two stimuli were delivered: fiber 1 responded to both, fiber 2 responded to only the first stimulus. Vertical calibration for $b, c$, and $d$ is $40 \mathrm{mV}$.

normally by the giant fiber, but the DLM is not. In Table I the latencies of $g f A^{1}$ and wild-type are compared. The DLM response latency is abnormally long and variable (mean latency $=3.21 \pm 0.94 \mathrm{msec}$ versus $1.30 \pm 0.09$ for wild-type). In addition, the DLM can be driven by the giant fiber only at abnormally low frequencies. The giant fiber output to the DLM at stimulating frequencies of 2 $\mathrm{Hz}$ is shown in Figure $5 b$. It can be seen that at this frequency the DLM cannot follow 1:1. In comparison, the DLM in wild-type flies can be driven by the giant fiber at frequencies exceeding $100 \mathrm{~Hz}$ (Tanouye and Wyman, 1980).

The abnormal DLM response in the mutant is not due to defects in motor neuron conduction or neuromuscular transmission. Figure $5 c$ shows a $g f A^{1}$ DLM fiber response to stimulation of its motor neuron at $50 \mathrm{~Hz}$. The response latency is normal (Table $\mathrm{I}$ ), and the maximum following frequency was found to be, like wild-type, in excess of $100 \mathrm{~Hz}$. This shows that the defect in $g f A^{1}$ must lie centrally, either in the giant fiber output to the PSI or in the PSI itself.

If this site of lability were exclusively the GF-PSI synapse, one would expect that for each stimulus the PSI would be either driven or not. Since all the DLM fibers are driven by the PSI, they should all fail together when the PSI fails. However, this was found not to be the case.
Figure $5 d$ shows responses in two ipsilateral DLM fibers evoked by brain stimulation of the giant fibers. Of the two stimuli delivered, DLM fiber 1 responded to both, and DLM fiber 2 responded only to one. Other pairs of DLM units were recorded from, and in all cases $(n=10)$ the fibers failed independently.

Genetics of gfA. gf $A^{1}$ maps proximally to forked. 'The physiological phenotype of $g f A^{2}$ homozygotes or gf $A^{1}$ / $g f A^{2}$ heterozygotes is indistinguishable from $g f A^{1}$. Thus, $g f A^{1}$ and $g f A^{2}$ are allelic. The physiological phenotype of gf $A^{1} / D f(1) J A 27$ females is indistinguishable from $g f A^{1}$ hemizygous males or homozygous females, and on this basis the mutation is located cytologically between bands $18 \mathrm{~A} 5$ and $18 \mathrm{Dl}$.

\section{Discussion}

$G F$-mediated escape response. The giant fiber circuit can be activated by three different means: intracellular stimulation of the giant fiber, extracellular stimulation of the giant fiber in the brain, and a light-off visual stimulus. In each case the pattern of response observed in the GF, TTM, and DLM is indistinguishable from the other two. Intracellular stimulation of the giant fibers demonstrates that giant fiber activation, by itself, is sufficient to elicit the characteristic pattern of activity in the muscles. It is very likely that giant fiber activation 
is also necessary for elicitation of the response. It was never possible to drive the muscles in the GF-like pattern unless a GF spike was also elicited; for each mode of stimulation the threshold for GF activation was always the same as for activation of the TTM and DLM spikes at the appropriate latencies. By capitalizing on the finding that this circuit mediates a visually induced escape response, flies can readily be screened behaviorally for possible defects in the pathway. Among the behavioral mutants selected in the screen are those which have abnormal giant fiber motor output.

bendless. The electrophysiological evidence on ben mutants shows that the GF is present, is capable of carrying spikes, and drives the DLM normally. We also know, from the motor neuron stimulation, that the TTM neuromuscular junction and muscle are functioning normally. Thus, it can be concluded that the defect in bendless lies in the transmission between the GF and the TTM motor neuron. This conclusion is strengthened by an anatomical study of the GF in bendless showing that the GF extends to the region of its normal synapse with the PSI, but does not extend laterally to the region of normal synaptic contact with the TTM motor neuron (Thomas and Wyman, 1982).

Whether the giant fiber in the mutant actually makes direct synaptic contact with the TTM motor neuron is not known. However, the long and variable latency of the mutant GF-to-TTM pathway and the failure of 1:1 transmission at rates over $1 \mathrm{~Hz}$ are features characteristic of polysynaptic chemical pathways, thus suggesting the absence of direct synaptic contact between the GF and TTM motor neuron. The slow driving of the TTM that remains in the mutant could be due to a pathway which is normally present but masked in wild-type by the fast pathway through the GF-TTM motor neuron electrical synapse. Alternatively, it could be due to aberrant synapses made by the GF in the mutant. The TTM can normally be driven by pathways other than the GF (see above), and abberant synapses might be made onto premotor neurons of these pathways.

passover. In passover flies the giant fiber is present and carries spikes. The TTM motor neuron is present and drives the muscle normally. Therefore, the abnormal driving of the TTM in pas is also due to disruption of transmission between the GF and the TTM motor neuron. This physiological defect has been shown to have a morphological correlate. In a study of the TTM motor neuron morphology in pas, Koto (1983; and cited in Thomas and Wyman, 1983) found that the TTM motor neuron branch, which normally extends to the point of synaptic contact with the ipsilateral GF, extends past this point, crosses the midline, and enters the contralateral neuromere. The GF morphology is normal in pas flies (Thomas, 1980).

The GF can still drive the TTM with an abnormally long latency and greatly reduced following frequency. Unlike bendless, where the TTM latency is 1.2 to 2.8 msec longer than normal, passover flies have TTM latencies only 0.4 to $1.0 \mathrm{msec}$ longer than wild-type. Therefore, it is possible that the GF still makes direct synaptic contact with the TTM motor neuron, but that synaptic transmission is malfunctioning in some manner to pro- duce the abnormally long latency and low following frequency. Alternatively, the direct synaptic connection between the GF and TTM motor neuron may be absent and the GF may drive the TTM motor neuron via a polysynaptic pathway as discussed above for ben.

Since the DLM neuromuscular junctions are normal in pas, the lack of DLM driving by the GF must be due to defects in the transmission between either the GF and the PSI or between the PSI and DLM motor neurons (or both). We have no evidence to distinguish between these possibilities.

gfA. Since the DLM neuromuscular junctions function normally and the GF carries spikes (and drives the TTM normally), the defect in $g f A$ lies in either the transmission between the GF and the PSI or between the PSI and DLM motor neurons. It is possible that some of the connections in the pathway are simply not made. In this case the abnormal driving of the DLMs would be mediated by another, perhaps aberrant, pathway between the GF and the DLM motor neurons. The long and variable latency and low following frequency suggest the presence of polysynaptic driving. Alternatively, connectivity may be intact in the mutant and the synapses malfunction to produce the abnormal DLM response. In this case, the defect should reside in PSI-to-DLM motor neuron transmission since the ipsilateral DLM fibers (all driven by the PSI) fail independently.

Genes and neuronal connectivity. The first step in searching for genes that are responsible for the specificity of neuronal connection is to determine if mutations exist which differentially disrupt specific synapses in a pathway. We have found that such mutations do exist. The GF has two output paths: to the TTM and to the DLM. One mutation (ben) disrupts only the former pathway, one mutation ( $g f A$ ) disrupts only the latter pathway, and the third mutation (pas) disrupts both. Of the two mutations which affect the same synapse (GF-TTM) one clearly alters the morphology of the presynaptic neuronal branch where this synapse is located, while the other mutation clearly affects the relevant branch of the postsynaptic neuron (Thomas and Wyman, 1982; Koto, 1983).

Before drawing any conclusions about the specificity or function of the affected genes, several lines of further research are necessary. Since we have only studied a very restricted piece of the nervous system, the mutations may have effects elsewhere. The acquisition and analysis of more extreme alleles at the loci might also reveal additional defects. However, the finding that the physiological phenotypes of ben/Df, pas/Df, and gfA/Df are, within present limits of analysis, indistinguishable from their homozygous counterparts suggests that these alleles are not leaky in the sense that gene function is merely reduced. Instead, gene function may be completely obliterated, and these mutations may be the most extreme alleles at their respective loci.

\section{References}

Campos-Ortega, J. A., and F. Jimez (1980) The effects of Xchromosome deficiencies on neurogenesis in Drosophila. In Development and Neurobiology of Drosophila, O. Siddiqi, P. Babu, L. M. Hall, and J. C. Hall, eds., pp. 201-222, Plenum Press, New York. 
Costello, W. J., and J. B. Thomas (1981) Development of thoracic muscles in muscle-specific mutant and normal Drosophila melanogaster. Soc. Neurosci. Abstr. 7: 543.

Grigliatti, T., L. Hall, R. Rosenbluth, and D. T. Suzuki (1973) Temperature-sensitive mutations in Drosophila melanogaster. XIV. A selection of immobile adults. Mol. Genet. 120: 107-114.

Hall, J. C. (1982) Genetics of the nervous system in Drosophila. Q. Rev. Biophys. 15: 223-479.

Harris, W. A., W. S. Stark, and J. A. Walker (1976) Genetic dissection of the photoreceptor system in the compound eye of Drosophila melanogaster. J. Physiol. (Lond.) 256: 415-439.

Heisenberg, M., R. Wonneberger, and R. Wolf (1978) optomotorblind ${ }^{\mathrm{H} 31}$ - a Drosophila mutant of the lobula plate giant neurons. J. Comp. Physiol. 124: 287-296.

Kaplan, W. D., and W. E. Trout (1974) Genetic manipulation of an abnormal jump response in Drosophila. Genetics 77: 721-739.

Kelly, L. E. (1974) Temperature-sensitive mutations affecting the regenerative sodium channel in Drosophila melanogaster. Nature 248: 166-168.

King, D. G., and R. J. Wyman (1980) Anatomy of the giant fiber pathway in Drosophila. I. Three thoracic components of the pathway. J. Neurocytol. 9: 753-770.

Koto, M. (1983) Morphology of giant fiber system neurons in wild-type and mutant Drosophila melanogaster. Ph.D. thesis, Yale University, New Haven, CT.

Koto, M., M. A. Tanouye, A. Ferrus, J. B. Thomas, and R. J. Wyman (1981) The morphology of the cervical giant fiber neuron of Drosophila. Brain Res. 221: 213-217.

Lewis, E. B., and F. Bacher (1968) Methods of feeding ethyl methane sulfonate (EMS) to Drosophila males. Drosophila Inform. Serv. 43: 193.

Lindsley, D. L., and E. H. Grell (1968) Genetic Variations of Drosophila melanogaster. Carnegie Institute Publication No. 627, Washington, D. C.

Meyerowitz, E. M., and D. R. Kankel (1978) A genetic analysis of visual system development in Drosophila melanogaster. Deve. Biol. 62: 112-142.

Mulloney, B. (1969) Interneurons in the central nervous system of flies and the start of flight. Z. Vergl. Physiol. 64: 243-253.

Power, M. E. (1943) The effect of reduction in numbers of ommatidia upon the brain of Drosophila melanogaster. J. Exp. Zool. 94: 33-71.

Salkoff, L., and L. Kelly (1978) Temperature induced seizure and frequency-dependent block in a $t s$ mutant of Drosophila. Nature 273: 156-158.

Salkoff, L., and R. J. Wyman (1981) Genetic modification of potassium channels in the Drosophila Shaker mutants. Nature 293: 228-230.

Schalet, A., and G. Lefevre (1976) The proximal region of the X-chromosome. In Genetics and Biology of Drosophila, M. Ashburner and E. Novitski, eds., pp. 847-902, Academic Press, Inc., New York.

Siddiqi, O., and S. Benzer (1976) Neurophysiological defects in temperature-sensitive paralytic mutants of Drosophila melanogaster. Proc. Natl. Acad. Sci. U. S. A. 73: 3253-3257.

Stewart, W. W. (1978) Functional connections between cells as revealed by dye-coupling with a highly fluorescent napthalimide tracer. Cell 14: 741-759.

Strausfeld, N. J., and R. N. Singh (1980) In Development and Neurobiology of Drosophila, O. Siddiqi, P. Babu, L. M. Hall, and J. C. Hall, eds., pp. 267-291, Plenum Press, New York.

Suzuki, D. T., T. Grigliatti, and R. Williamson (1971) Temperature-sensitive mutations in Drosophila. VII. A mutation (para-ts) causing reversible adult paralysis. Proc. Natl. Acad. Sci. U. S. A. 68: 890-893.

Tanouye, M. A., and R. J. Wyman (1980) Motor outputs of the giant nerve fiber in Drosophila. J. Neurophysiol. 44: 405-421.

Thomas, J. B. (1980) Mutations affecting the giant fiber system of Drosophila Soc. Neurosci. Abstr. 6: 742.

Thomas, J. B., and R. J. Wyman (1982) A mutation in Drosophila alters normal connectivity between two identified neurons. Nature 298: 650-651.

'Thomas, J. B., and R. J. Wyman (1983) Normal and mutant connectivity between identified neurons in Drosophila. Trends Neurosci. 6: 214-219.

Wu, C. -F., and B. Ganetsky (1980) Genetic alteration of nerve membrane excitability in temperature-sensitive paralytic mutants of Drosophila melanogaster. Nature 286: 814-816. 